\title{
Associations Between Age, Psychosocial Work Conditions, Occupational Well-Being, and Telomere Length in Geriatric Care Professionals
}

\author{
A Mixed-Methods Study
}

\author{
Caroline Chmelar, MD, Rudolf A. Jörres, PhD, Angelika Kronseder, CTA, Andreas Müller, PhD, \\ Dennis Nowak, MD, and Matthias Weigl
}

\begin{abstract}
Objective: We identified associations between age, psychosocial work characteristics, occupational well-being, and-as a measure of biological age-leukocyte telomere length in geriatric care professionals. Methods: This is a multisource study of self-reports on psychosocial work characteristics, standardized physician's evaluations of health, and relative telomere length measures of peripheral blood leukocytes. We included 141 geriatric care professionals. Telomere length was assessed by an improved polymerase chain reaction (PCR)-based method. Results: Increased depersonalization was associated with shorter telomeres. Their association with age was not moderated by psychosocial work conditions. There was, however, a significant three-way interaction of social support and work ability with the agetelomere association. Additionally, social support and adverse general health moderated the age-telomere length relationship. Conclusions: A supportive work environment and work-related health may influence the association between age and telomere length.
\end{abstract}

\section{OBJECTIVE}

$\mathrm{F}$ ew topics in Occupational Medicine spurred so much attention during past years than work-related factors that contribute to successful aging of employees. Health and work conditions have a key role for work functioning, including job retention, and premature retirement. ${ }^{1,2}$ Design of healthy work environments for ageing workers contributes to productivity and welfare of societies.

Our study specifically focuses on the associations between psychosocial work characteristics, occupational well-being, and telomere length in geriatric care professionals. Telomere length has drawn increased attention in Occupational Medicine as indicator for biological aging. ${ }^{4-6}$ Accelerated telomere shortening can be caused by oxidative stress and is also associated with high psychological stress. ${ }^{7}$ Shorter leukocyte telomeres have been detected in smokers, subjects with cardiac risk factors, obese individuals, ${ }^{8-13}$ and other health disorders. Correspondingly, a shortened leukocyte telomere length

From the Institute and Clinic for Occupational, Social and Environmenta Medicine, Ludwig-Maximilians-University Munich (Dr Chmelar, Dr Jörres, Ms Kronseder, Dr Nowak, Mr Weigl); Institute for Occupational, Social, and Environmental Medicine, Heinrich-Heine-University Düsseldor (Dr Müller), Germany.

Fundings: This study was partly funded by the Munich Centre for Health Sciences (MC-Health).

Authors Chmelar, Jörres, Kronseder, Müller, Nowak, and Weigl have no relationships/conditions/circumstances that present potential conflict of interest.

The JOEM editorial board and planners have no financial interest related to this research.

Address correspondence to: Caroline Chmelar, MD, Institute and Clinic for Occupational, Social and Environmental Medicine, Ludwig-MaximiliansUniversity Munich, Ziemssenstrasse 1, 80336 Munich, Germany (caroline. chmelar@med.uni-muenchen.de).

Copyright (C) 2017 American College of Occupational and Environmental Medicine

DOI: $10.1097 / \mathrm{JOM} .0000000000001102$

\section{Learning Objectives}

- Become familiar with recent occupational medicine studies of telomere lengthening as an indicator of biological aging.

- Summarize the new findings on associations of telomere length with psychosocial work conditions and occupational well-being in geriatric care professionals.

- Discuss the implications for design of healthy work environments to promote successful aging of employees.

is associated with increased risks for coronary heart disease, stroke, myocardial infarction, and type 2 diabetes mellitus. ${ }^{12,14}$

Only few studies, however, have examined associations

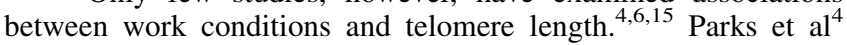
reported that women with fulltime work had significantly shorter telomeres than non-employed women. Among the variety of behavioral factors, lack of sleep and recovery are associated with shorter telomere lengths. ${ }^{5,16,17}$ Nonetheless, other studies found no associations of telomere length with employment status, job strain categories, and occupational categories, as well as levels of adverse psychosocial exposures. ${ }^{15}$

These discrepancies might be partially due to the neglect of the interplay between age, psychosocial work characteristics, workrelated health, and telomere length. Psychosocial work characteristics have been shown to be influential for health at work as well as functioning across the work life span. ${ }^{18,19}$ To the best of our knowledge, there is no study that has investigated the joint associations of time pressure, job autonomy, and social support with telomere length. All three have been identified as major determinants for functioning at work across all ages. ${ }^{1,20}$ Geriatric care is a profession with particularly high job demands, and geriatric care organizations face high turnover rates and significant difficulties in recruiting. ${ }^{21-23}$ Geriatric care professionals are exposed to several stressors such as time pressure, workload, lack of social support, and shiftwork. ${ }^{21,22,24}$

In respect to the association between work related health and telomere length, Ahola et $\mathrm{al}^{6}$ observed shorter telomeres in employees with emotional exhaustion-one core component of burnout. Their study did not consider depersonalization which is considered the second core component of burnout. ${ }^{21,25}$ Therefore, investigations are necessary that consider both burnout components and their potential association with telomere length. Moreover, we are not aware of studies that investigated the association between telomere length and personal characteristics of successful ageing — such as work ability and general health. Work ability is defined as the individual physical and psychological capacity to perform work and is known to be linked to age. ${ }^{1,26-28}$ Poor work ability is associated with reduced physical and mental well-being, emotional exhaustion, and intention to leave the job. ${ }^{27-30}$ In the field of care professionals it has been repeatedly shown that the work ability of nurses decreases with age. ${ }^{27,28}$ Among nurses, low work ability is related to low mental and physical 
well-being, emotional exhaustion, and the intention to leave the nursing job. ${ }^{27-30}$

\section{Study Objectives}

In order to extend the knowledge about the association between a healthy work environment and physiological indicators of ageing, we sought to identify health- and work-related conditions that affect the known relationship between age and telomere length, as this would suggest a biological effect of these conditions. Our first aim was to assess the effects of mental and physical components of occupational well-being on telomere length. Our second aim was to identify associations between psychosocial work characteristics and telomere length. Here, we sought to explore whether the association between age and telomere length is jointly moderated by the employees' psychosocial work conditions and occupational well-being.

\section{METHODS}

\section{Design, Setting, and Procedure}

We set up a cross-sectional study with multisource data consisting of self-reports, expert physician evaluations based on physical examinations, and employees' bio samples. The study was approved by the Ethics Committee of the Medical Faculty of Ludwig-Maximilians-University of Munich (No. 99-15).

Employees from six geriatric care homes in Southern Germany were surveyed and examined. The survey and examinations were conducted on-site during the regular health examinations by an occupational physician. All employees were informed prior to the study. Participation was voluntary and employees provided written consent prior to handing out the survey. The participants filled out a questionnaire survey and provided a blood sample for telomere measurement. After that, the occupational physician performed a physical examination.

\section{Study Sample}

Overall, 212 employees were eligible, while 11 (5.19\%) refused participation and $60(28.30 \%)$ missed the scheduled appointment, for example, due to vacation, illness, or short-term shift changes. Overall, 141 professionals were finally included (response rate: $67.2 \%$ ). The sample included mainly geriatric care professionals but also kitchen staff and cleaning personnel. Geriatric care nurses in all facilities worked in a three-shift schedule, whereas the kitchen and cleaning personnel routinely worked in a two-shift schedule.

\section{Measures}

\section{Psychosocial Work Characteristics}

Three psychosocial work characteristics were measured with a validated and well-established self-report instrument for work analysis of care work environment ${ }^{31}$ : (1) time pressure was measured with three items (eg, "I often have too much work to do at once"). (2) Job autonomy was assessed with four items (eg, "My work allows for decisions on which methods I pursue"). Both scales ranged from $1=$ "no, not at all" to $5=$ "yes, to a great extent." (3) Social support at work was measured with two questions regarding sources of support ("To what extent do you receive support from your supervisor?/.. . your colleagues?"). The answer scale ranged from $1=$ "not at all" to $4=$ "to a great extent."

\section{Burnout}

Respondents' emotional exhaustion and depersonalization were measured with the German version of the Maslach-Burnout-Inventory. ${ }^{25,32}$ Emotional exhaustion was assessed with a three-item scale (eg, "I feel burned out from my work"), depersonalization also with three items (eg, "I don't really care what happens to some of my clients"). A summary frequency scale was applied from $1=$ "never" to $6=$ "very often." 32

\section{Physician Examination Data}

All participants took part in standard clinical examinations for health and the assessment of medical history and sociodemographic data conducted by an occupational physician. The following data were obtained: sex, age, height, weight, smoking status, shift, working hours per week, duration of employment, existing of severe disability, physical activity, cardiac risk factors, diabetes mellitus, and medication.

Work ability was evaluated with an abbreviated version of the German Work Ability Index (WAI). ${ }^{33}$ Previous work has indicated that shortened, abbreviated measures of the WAI are highly correlated with the overall WAI-score and also are predictive for well-being. ${ }^{30}$ Three items of two WAI-dimensions were applied. The first item covers current work ability compared with that of the individual's lifetime best (range $0=$ "totally no work ability" to $10=$ "currently best work ability ever"; scale values were subsequently divided by two to align with the other two following WAI items). The two other items of the second WAI dimension measure the perceived work ability in relation to physical and psychological job demands. ${ }^{33}$ The answers were based on 5-point Likert scales ranging from $1=$ "very poor" to $5=$ "very good." Adverse general health was also evaluated by the physician. The respective interview question was "How would you rate your health status in general?", and the options for answer ranged from $1=$ "excellent" to $5=$ "bad."

\section{Telomere Length}

Samples of peripheral venous ethylenediaminetetraacetic acid blood were taken during the examination and kept in aliquots frozen at $-80^{\circ} \mathrm{C}$ until analysis. DNA was isolated using the QIAamp DNA Blood Mini Kit (Qiagen, Hilden, Germany) according to the manufacturer's protocol. For telomere length determination a combination of the monochrome multiplex real-time quantitative polymerase chain reaction methods described by Cawthon $^{34}$ and Tackney ${ }^{35}$ was used, with some modifications based on experiences gained in past analyses within the institute's laboratory. In brief, 96-well plates were run with $20 \mu \mathrm{L}$ reaction volume in the CFX96 Real-Time Polymerase Chain Reaction (PCR) Detection System (Bio-Rad, Munich, Germany). Telomere (telg/telc) and albumin (albugcr2/albdgcr2) primers were chosen in final concentrations of telg/telc $200 \mathrm{nM}$ each and albugcr2/albdgcr2 $100 \mathrm{nM}$ each. ${ }^{35}$ Five times HOT FIREpol HRM Mix (no ROX) (Solis Biodyne) was used as master mix according to manufacturer's protocol. ${ }^{36}$ The final temperature set-up was as following. Stage 1: 15 minutes at $95{ }^{\circ} \mathrm{C}$; Stage 2: two cycles of 15 seconds at $95^{\circ} \mathrm{C}, 30$ seconds at $49^{\circ} \mathrm{C}$; Stage 3: 32 cycles of 15 seconds at $95^{\circ} \mathrm{C}, 20$ seconds at $60^{\circ} \mathrm{C}, 20$ seconds at $72^{\circ} \mathrm{C}$ with signal acquisition, 30 seconds at $84^{\circ} \mathrm{C}$, and 20 seconds at $85^{\circ} \mathrm{C}$ with signal acquisition. A melting curve analysis was included for each realtime quantitative polymerase chain reaction run. The standard curves in the range of 2.5 to $80 \mathrm{ng}$ reference DNA were created by serial dilutions. Efficiency values for the standard curves from $91 \%$ to $111 \%$ and $R^{2}$ is greater than or equal to 0.99 were accepted. Relative telomere length was expressed as telomere/albumin ratio. ${ }^{34}$

\section{Statistical Analyses}

Descriptive statistics including frequencies, means, and standard deviations were used to describe respondent demographics, survey responses, and examination results. Scale reliabilities were tested applying Cronbach $\alpha$ as indicator. All scales showed very good to good internal consistencies (cf., Table 1). In correlation analyses we examined bivariate associations among demographic data and study variables. For our main objectives, hierarchical linear regression 
TABLE 1. Prevalence of Sociodemographic Characteristics, Occupational Well-Being and Telomere Length

\begin{tabular}{|c|c|c|c|}
\hline & $\begin{array}{l}\text { Scale/Scale } \\
\text { Range }\end{array}$ & $\begin{array}{c}\text { Reliability } \\
\text { (CA) }\end{array}$ & $\begin{array}{c}\text { Prevalence } \\
M \pm \mathrm{SD} / \boldsymbol{n}(\%)\end{array}$ \\
\hline \multicolumn{4}{|l|}{ Sociodemographic characteristics } \\
\hline Age & (yrs) & & $44.14 \pm 12.35$ \\
\hline Weekly working Time & (h/week) & & $37.23 \pm 7.72$ \\
\hline Shiftwork & (no/yes) & & $117(83.0)$ \\
\hline Nicotine consumption & (packyears) & & $5.53 \pm 9.64$ \\
\hline Diagnosis of diabetes & (no/yes) & & $3(2.1)$ \\
\hline Cardiac risk group & (no/yes) & & $59(41.8)$ \\
\hline \multicolumn{4}{|l|}{ Psychosocial work conditions } \\
\hline Time pressure & {$[1,5]$} & 0.88 & $3.23 \pm 1.06$ \\
\hline Job autonomy & {$[1,5]$} & 0.87 & $3.20 \pm 1.06$ \\
\hline Social support & {$[1,4]$} & 0.59 & $3.06 \pm .68$ \\
\hline \multicolumn{4}{|l|}{ Occupational well-being } \\
\hline Burnout: emotional exhaustion & {$[1,6]$} & 0.92 & $3.81 \pm 1.25$ \\
\hline Burnout: depersonalization & {$[1,6]$} & 0.72 & $1.75 \pm .91$ \\
\hline Work ability & {$[1,5]$} & 0.82 & $4.21 \pm .76$ \\
\hline Adverse general health & {$[1,5]$} & - & $2.14 \pm .85$ \\
\hline \multicolumn{4}{|l|}{ Biological marker } \\
\hline Telomere length & & - & $0.96 \pm .17^{*}$ \\
\hline
\end{tabular}

Notes: $n=141$, M, mean; SD, standard deviation; CA, Cronbach $\alpha$.

${ }^{*}$ Telomere length expressed as relative units (see methods).

analyses were applied to determine the crude and adjusted associations between the determinant variables (ie, age, work characteristics, individual health) and the outcome variable, that is, telomere length. All determinants were included as continuous variables and were standardized to reduce collinearity. Tests of moderation hypotheses were based on interaction terms of the determinant variables that were included stepwise into the regression model. All statistical analyses were performed using SPSS 23.0 (IBM Inc., Chicago, IL). Statistical significance was assumed for $P<0.05$.

\section{RESULTS}

\section{Descriptive Statistics}

\section{Sociodemographic Data}

We obtained data from $N=141$ professionals of six geriatric care homes. The sample comprised 112 women (79.4\%). The majority had a full-time contract $(n=119,84.4 \%)$, and 18 participants worked part time $(13.1 \%)$. The average professional tenure was $M=22.30$ years (standard deviation $[\mathrm{SD}]=1.94$, range 0.5 to 50 years), the average working time per week $M=37.23$ hours $(\mathrm{SD}=7.7$, range 7 to 45 hours). Overall, 117 employees $(84.6 \%)$ were on shiftwork schedule (2- or 3-shift system) and $23(16.4 \%)$ reported no shiftwork obligations.

\section{Clinical Examination}

Fifty nine participants $(41.8 \%)$ were classified into a group with elevated cardiac risks (hypercholesterinemia, hypertension, diabetes, nicotine consumption, coronary heart disease/myocardial infarction in first line family members) and three participants $(2.1 \%)$ had diagnosed type 2-diabetes. $67.4 \%$ of the surveyed professionals were never-smokers (ie, 0 pack-years, py), $11.6 \%$ smoked between 1 and 10 py, $13.5 \%$ between greater than 10 and 20 py, and $8.5 \%$ greater than 20 py. BMI characteristics were $M=25.65$ ( $\mathrm{SD}=4.30$ ).

\section{Blood Samples}

The average leucocyte count was $M=7.08 \times 10^{9} / \mathrm{L}$ $(\mathrm{SD}=1.52)$, and the proportion of eosinophil granulocytes
$M=0.15 \%$ (ie, share of the total granulocyte number, $\mathrm{SD}=0.11$ ). No participant had less than $4 \times 10^{9} / \mathrm{L}$ leucocytes, three showed greater than $11 \times 10^{9} / \mathrm{L}$ leucocytes $(0.7 \%)$. The correlations with age were not significant: $r=-0.04(P=0.63)$ for leucocytes, and $r=-0.08(P=0.32)$ for eosinophils.

\section{Study Variables} Table 1.

The mean values of our study variables are reported in

\section{Associations of Study Variables and Telomere Length Outcome}

Next, we tested for bivariate associations between sociodemographic characteristics and telomere length in order to reveal whether confounding effects were present. Table 2 reports the results.

\section{Two Identified Effects Deserve Consideration}

Although not significant, nicotine consumption tended to be associated with reduced telomere lengths. We obtained a significantly negative relationship between employee's age and telomere length.

Subsequently, we tested for associations between work characteristics, well-being, and health at work, and telomere length outcomes, respectively. Crude bivariate associations were determined and then adjusted for confounders that showed meaningful associations in the previous analyses (cf., Table 2). Table 3 reports the associations found by this approach.

High depersonalization was significantly associated with reduced telomere length after controlling for sociodemographic confounders (cf., Table 3). Moreover, there was a significant relationship between adverse health and telomere length such that inferior health status was linked to reduced telomere length. This association remained however not significant after controlling for sociodemographic confounders (cf., Table 3).

In the next step, potential work- or health-related moderators for the relationship between age and telomere length were examined. First, we checked the assumption that work characteristics moderate this relationship. There was no interaction between age and psychosocial work characteristics, all respective interaction terms were non-significant; for job autonomy: $B=0.02,95 \% \mathrm{CI}$ -0.01 to $0.05, P=0.17$, for time pressure: $B=0.01,95 \% \mathrm{CI}-0.02$ to $0.04, P=0.54$; for social support: $B=0.01,95 \% \mathrm{CI}-0.02$ to $0.03, P=0.70$

In the next step, we explored if the interaction between time pressure and job autonomy as well as time pressure and social support influence telomere length. All analyses were controlled for

TABLE 2. Associations Between Sociodemographic Characteristics and Telomere Length

\begin{tabular}{lcc}
\hline & \multicolumn{2}{c}{ Outcome: Telomere Length } \\
\cline { 2 - 3 } & \multicolumn{1}{c}{ Associations } & $\begin{array}{c}\text { Significance } \\
\text { Testing }\end{array}$ \\
\cline { 2 - 3 } & $\boldsymbol{B}[\mathbf{9 5 \%} \mathbf{C I}]$ & {$[\boldsymbol{P}]$} \\
\hline Sociodemographic characteristics & & \\
Working time (h/week) & $0.00[0.00,0.01]$ & 0.09 \\
Shiftwork (no/yes) & $-0.02[-0.10,0.06]$ & 0.62 \\
Nicotine consumption (no/yes) & $0.00[-0.01,0.00]$ & 0.07 \\
Diabetes diagnosis (no/yes) & $-0.12[-0.32,0.08]$ & 0.22 \\
Cardiac risk (no/yes) & $-0.03[-0.09 ; 0.03]$ & 0.31 \\
Age (in yrs) & $\mathbf{- 0 . 0 6}[-\mathbf{0 . 0 9} ;-\mathbf{0 . 0 3}]$ & $<\mathbf{0 . 0 1}$ \\
\hline
\end{tabular}

Notes: $N=141$, Bivariate linear regression analyses, $B$, non-standardized regression coefficient, 95\% CI: 95\% confidence interval, bolded if $P<0.05$. 
TABLE 3. Associations Between Work Characteristics, Well-Being and Telomere Length

\begin{tabular}{|c|c|c|c|c|}
\hline & \multicolumn{4}{|c|}{ Outcome: Telomere Length } \\
\hline & $B[95 \% \mathbf{C I}]$ & $\boldsymbol{P}$ & $B[95 \% \mathbf{C I}]$ & $P$ \\
\hline \multicolumn{5}{|l|}{ Psychosocial work characteristics } \\
\hline Autonomy & $-0.00[-0.03,0.03]$ & 0.97 & $-0.01[-0.04,0.02]$ & 0.66 \\
\hline Time pressure & $0.01[-0.02,0.04]$ & 0.58 & $0.01[-0.02,0.04]$ & 0.61 \\
\hline Burnout: emotional exhaustion & $0.00[-0.03 ; 0.03]$ & 0.91 & $0.00[-0.02,0.03]$ & 0.76 \\
\hline Burnout: depersonalization & $-0.02[-0.05,0.01]$ & 0.21 & $-0.03[-0.06,-0.01]$ & $\mathbf{0 . 0 2}$ \\
\hline Work ability & $0.02[-0.01,0.04]$ & 0.27 & $-0.03[-0.06,0.00]$ & 0.08 \\
\hline Adverse general health & $-0.03[-0.06,0.00]$ & 0.04 & $0.00[-0.03,0.03]$ & 0.99 \\
\hline
\end{tabular}

Notes: $n=141$. Tests for associations were computed with standardized variables; adjusted for age, working time, shiftwork, nicotine consumption, cardiac risk, and diabetes; bolded if $P<0.05$.

$B$, non-standardized regression coefficient; $95 \% \mathrm{CI}$ : $95 \%$ confidence interval.

all above described confounders including age. We did not observe significant moderation on telomere length; neither for job autonomy $\times$ time pressure interaction $(B=0.00,95 \% \mathrm{CI}-0.03$ to 0.03 , $P=0.94)$, nor for the social support $\times$ time pressure interaction $(B=0.01,95 \%$ CI -0.02 to $0.04, P=0.49)$.

In the final step potential three-way interactions such that work characteristics as well as mental well-being at work moderate the association between age and telomere length were explored. Three-way interactions with age, both burnout components (emotional exhaustion, depersonalization), and all three job characteristics separately, that is, time pressure, job autonomy, and social support were included. There were no significant moderations. We also explored potential interactions between age, work ability, and all three job characteristics taken separately. There was a significant three-way interaction of age, social support, and work ability $(B=-0.05,95 \% \mathrm{CI}-0.08$ to $-0.02, P=0.001)$ which is graphically depicted in Fig. 1. An additional test for slope differences showed that in employees with high social support and low work ability the association between age and telomere length was significantly different from employees with low social support as well as from professionals with high work ability and high social support (all $P<0.01$ ). No significant results were observed for the impact of time pressure or job autonomy together with age and work ability.

Lastly, we explored potential three-way interactions between age, adverse general health, and the three job characteristics. A significant effect was observed for the interplay of age, adverse



FIGURE 1. Significant three-way interaction of social support and work ability for the association between age and telomere length. general health, and social support $(B=0.04,95 \% \mathrm{CI} 0.01$ to 0.07$)$ as shown in Fig. 2. Similar to the interaction above described, the association between age and telomere length was significantly different in employees with high social support and inferior health. For time pressure and job autonomy, no significant moderation effects with adverse health on the relationship between age and telomere length were observed.

\section{DISCUSSION}

The maintenance of functioning across the work life span while offsetting the onset and degree of decline of age-related losses in high demanding jobs has become of fundamental importance in occupational medicine. An understanding of the factors required for successful aging in the workplace requires elaboration of the work context, employee's individual conditions, and their mutual relationships with age including direct biological correlates of aging. In line with this, our multisource study aimed to determine associations between age, psychosocial work conditions, occupational well-being, and telomere length in care professionals. This study highlights an important occupational health priority, as workers caring for the aging population are themselves aging. Our results contribute in different ways to the current knowledge base on successful aging in demanding jobs.

First, we expand the growing but still very limited knowledge base on telomere length as a biomarker in occupational medicine. This marker is suggested to indicate work-related aging and

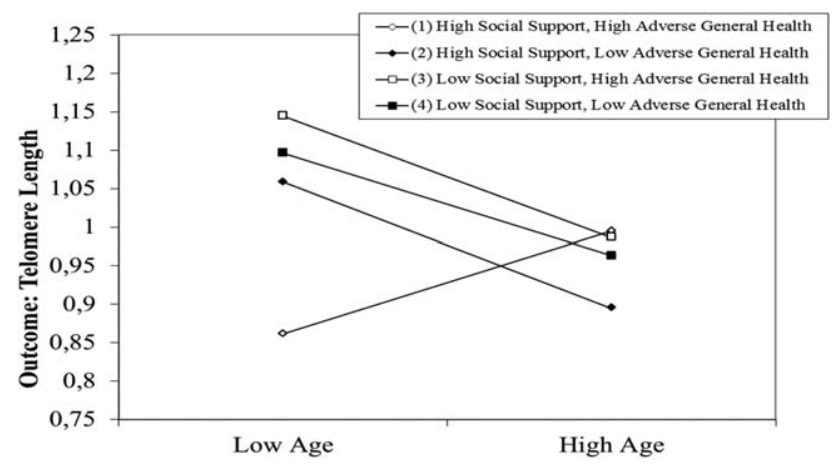

FIGURE 2. Significant three-way interaction of social support and adverse general health for the association between age and telomere length. 
environmentally mediated processes. ${ }^{4-6}$ Telomeres are a major predictor of cell functioning and eventually cellular senescence, and telomere length is a key indicator of biological aging. ${ }^{37,38}$ The significant association between employee's age and telomere length underlined the validity of the PCR method and suggests that the sample size was sufficient for our analyses.

Consistent with previous studies we observed that specific individual sociodemographic as well as behavioral risk factors tended to be associated with telomere length. Specifically, employees with increased cardiac risk factors had shorter telomeres than employees without these risk factors. ${ }^{9,10,14,39,40}$ The same was true for employees with diagnosed type 2 diabetes mellitus ${ }^{14,41}$ despite the fact that this group was very small in our sample $(n=3 ; 2.1 \%)$. Current smoking habits as well as a history of smoking had a major impact on telomere length, and smokers showed significantly shorter telomeres in line with previous findings. ${ }^{8,13}$ This is explained by the mechanism that smoking causes oxidative stress thereby increasing the rate of telomere erosion. ${ }^{42}$ The proportion of smokers in our sample was fairly representative of official census data for Germany. ${ }^{43}$ We did not find meaningful effects of shiftwork and working time. Previous studies showed inconsistent results in this regard. Liang et $\mathrm{al}^{5}$ found, that women with a history rotating night shifts work tended to have shorter telomeres, but the association was not significant. Parks et $\mathrm{al}^{4}$ found no association between work at night, rotating night shifts, and telomere length. Given to the relative homogeneity of our sample, future studies should draw upon professional samples with a larger variance of shiftwork and working time.

Second, our study suggests that increased states of burnout, and specifically depersonalization, are associated with reduced telomere length. Depersonalization is a core component of burnout and describes affective states of alienation and cynism in client care. Our study partly confirms findings of Ahola et $\mathrm{al}^{6}$ who reported a similar association between the burnout component emotional exhaustion and telomere length. At the same time our findings challenge these observations since we did not replicate this effect for emotional exhaustion. In contrast to Ahola et $a{ }^{6}{ }^{6}$ we examined a highly specialized professional sample of human service workers. The work of these professionals involves intensive direct contact with old, impaired, and occasionally disoriented clients what causes high work strain. Depersonalization represents the interpersonal context dimension of burnout and encompasses defensive and avoidant behaviors. ${ }^{44}$ Our findings suggest that this dysfunctional behavioral strategy is potentially a more important risk factor than emotional exhaustion. Our study demonstrates the importance to include both core components of burnout because they can have different associations with age-related outcomes. We further identified an association between adverse general health and telomere length, since employees with inferior health status were found to have shorter telomeres. This effect was not significant after adjusting for age and individual health conditions. This may be attributed to the dominant role of employee's diseases that are associated with reduced telomere length. ${ }^{45}$

The third and possibly foremost contribution of this study refers to person-environment interactions, specifically the interplay of psychosocial work characteristics and individual health conditions with the relationship between age and telomere length. To our knowledge this is the first study that explored interaction effects of psychosocial work conditions and employee health conditions on the age-related changes of this biomarker. Although these exploratory findings must be interpreted carefully, we deem that the identification of consistent three-way-interactions of age and social support with adverse health individual conditions, that is, diminished work ability as well as inferior general health, expands previous findings in this area. Consistent with other studies, our results revealed that work- and employee-related determinants had minor main effects but that the interaction of both with age showed a meaningful result. ${ }^{46}$ This fits well with the claim that the research in the field of work and aging should take more account of personenvironment interactions. ${ }^{18}$ Interestingly, among the three psychosocial work characteristics, there were no direct or indirect effects of time, pressure, and job autonomy on telomere length. One the other hand, our results emphasize the role of social support by colleagues and leaders in the course of successful ageing. In professionals with high social support but low work ability, the negative association between age and telomere length association was buffered. This effect was consistent with the role of adverse general health. It seems not farfetched to speculate that efficient social support at work is influenced by professionals' low work ability or poor general health. Accordingly, participants with low work ability or poor general health would have obtained more efficient support with a facilitating effect for ageing processes. This hypothesis generated from our observations appears worthwhile to be investigated in future studies.

Biological aging as indicated by telomere shortening and occurring as a result of age and environmental exposures may alter the employees' trajectories into later stages of work life. Our findings suggest that well-being at work and a supportive psychosocial work environment exert a joint effect on the association between chronological and biological aging. The study results call for future attempts to use telomere length as an indicator for the evaluation of work-related determinants of biological aging and the associated sequelae of functioning at work. ${ }^{46}$ This particularly applies to the joint analysis of psychosocial work characteristics and individual health conditions at work in relation to the biological marker telomere length.

\section{Limitations}

The findings of our study should be interpreted in the light of several limitations. Due to the cross-sectional design no causal inferences can be drawn. We used a relatively small sample of 141 professionals compared with previous studies on telomere length and occupational health. ${ }^{4,6}$ Although this may raise critics concerning the lack of statistical power (particularly for the identification of interaction effects) it should be noted that we investigated a rather homogeneous professional sample in contrast to similar investigations. ${ }^{4,6}$ Sample size was not established according prior statistical power analyses since we used an exhaustive convenience sample approach. We cannot exclude potential healthy-worker effects which might be relevant for age-related effects such that older persons with poor health status employed in age-critical workplaces quit jobs prematurely and are not included in studies such as the present one. ${ }^{29}$ Our findings call for longitudinal surveys that include different age cohorts. ${ }^{46}$ We only obtained information concerning the current state of psychosocial work characteristics but we cannot draw inferences about previous exposure to adverse psychosocial work environments. With detailed work-history data about the duration and extent of psychosocial stressors, the effects for telomere length outcomes might be better depicted. This applies also to shiftwork and working time information. Our data draw on selfreport evaluations thereby increasing the risk for spurious results due to self-report bias or age-related stereotypes. ${ }^{47}$ Notwithstanding, we relied on multisource data including self-reports, expertbased evaluation, and a biological sample (telomere length) to avoid common-method bias. Moreover, another study showed that selfreported data-collection provides a valid representation of the working conditions. ${ }^{31}$ Telomere length as biological marker of age has been subject to scientific discourse and critic, particularly with respect to its predictive accuracy. ${ }^{48}$ We acknowledge further that external validity of our results is limited since our data were derived from geriatric care professionals in Germany. Employee's work ability and adverse health measures were highly correlated and we obtained information on social support from two self-report 
items that are used for screening purposes. Therefore, we cannot specify which forms of social support (ie, affective or instrumental support) might be more relevant in our surveyed work setting. Moreover, other studies showed, that telomere length is associated with high oxidative stress, chronic illness, or high demands in ageing adults, such as informal caregiving outside the workplace or social status. ${ }^{7,9-11,49,50}$ As we did not collect data for family structure, educational background and financial resources, we cannot give data on the role of these factors and cannot state, that the age-dependence of telomere length only depends on the psychosocial factors analyzed in our study. Possibly the telomere length measured in our study also reflects a past history of accumulated exposures that are related to the cross-sectional measures which we employed. To clarify this, future studies should include both the actual state and the history of psychosocial factors and demographic characteristics in standardized questionnaires.

\section{Implications}

Regarding future research we consider our study as a first step that might inform future studies on appropriate approaches for the investigation of the interaction between chronological and biological ageing, psychosocial work environment, and individual health. Our survey included various measures and methods requiring resource-intensive assessments, but could be repeated with larger samples particularly for the identification of modest interaction effects. As practical implication, our findings underline the key role of social support at the workplace for biological aging and work ability. When workplaces are redesigned, these results should be taken into account.

\section{CONCLUSIONS}

Our findings suggest that individual health conditions at work and a supportive psychosocial work environment have a moderating influence on the association between chronological aging and telomere length as a marker of biological age, such that high social support buffers the negative association between chronological age and telomere lengths when subjective general health and work ability are low. Irrespective of the limitations of our study the results indicate potential applications regarding the use of telomere length as a biological measure of interactions between age and job demands in occupational medicine.

\section{ACKNOWLEDGMENTS}

This study was part of the doctoral thesis requirement of Caroline Chmelar (Ludwig-Maximilians-University Munich). The authors thank Dr. Tanja Lucke for her support in the establishment of PCR methods.

\section{REFERENCES}

1. van den Berg TI, Elders LA, de Zwart BC, Burdorf A. The effects of workrelated and individual factors on the work ability index: a systematic review. Occup Environ Med. 2009;66:211-220.

2. Siegrist J, Wahrendorf M, von dem Knesebeck O, Jurges H, Borsch-Supan A. Quality of work, well-being, and intended early retirement of older employees: baseline results from the SHARE Study. Eur J Public Health. 2007;17:62-68.

3. Attarchi M, Ghaffari M, Abdi A, et al. Assessment of the relationship between physical working conditions and different levels of work ability. Glob J Health Sci. 2014;6:213-220.

4. Parks CG, DeRoo LA, Miller DB, McCanlies EC, Cawthon RM, Sandler DP. Employment and work schedule are related to telomere length in women. Occup Environ Med. 2011;68:582-589.

5. Liang G, Schernhammer E, Qi L, Gao X, De Vivo I, Han J. Associations between rotating night shifts, sleep duration, and telomere length in women. PLoS ONE. 2011;6:e23462.

6. Ahola K, Siren I, Kivimaki M, et al. Work-related exhaustion and telomere length: a population-based study. PLoS ONE. 2012;7:e40186.

7. Sahin E, Depinho RA. Linking functional decline of telomeres, mitochondria and stem cells during ageing. Nature. 2010;464:520-528.
8. Valdes AM, Andrew T, Gardner JP, et al. Obesity, cigarette smoking, and telomere length in women. Lancet. 2005;366:662-664.

9. Brouilette SW, Whittaker A, Stevens SE, van der Harst P, Goodall AH, Samani NJ. Telomere length is shorter in healthy offspring of subjects with coronary artery disease: support for the telomere hypothesis. Heart. 2008;94:422-425.

10. Demissie S, Levy D, Benjamin EJ, et al. Insulin resistance, oxidative stress, hypertension, and leukocyte telomere length in men from the Framingham Heart Study. Aging cell. 2006;5:325-330.

11. Ma H, Zhou Z, Wei S, et al. Shortened telomere length is associated with increased risk of cancer: a meta-analysis. PLOS ONE. 2011;6:e20466.

12. Zhao J, Miao K, Wang H, Ding H, Wang DW. Association between telomere length and type 2 diabetes mellitus: a meta-analysis. PLOS ONE. 2013;8: e79993.

13. Latifovic L, Peacock SD, Massey TE, King WD. The influence of alcohol consumption, cigarette smoking, and physical activity on leukocyte telomere length. Cancer Epidemiol Prev Biomark. 2016;25:374-380.

14. D'Mello MJ, Ross SA, Briel M, Anand SS, Gerstein H, Pare G. Association between shortened leukocyte telomere length and cardiometabolic outcomes: systematic review and meta-analysis. Circ Cardiovasc Genet. 2015;8:82-90.

15. Fujishiro K, Diez-Roux AV, Landsbergis PA, Jenny NS, Seeman T. Current employment status, occupational category, occupational hazard exposure and job stress in relation to telomere length: the Multiethnic Study of Atherosclerosis (MESA). Occup Environ Med. 2013;70:552-560.

16. Starkweather AR, Alhaeeri AA, Montpetit A, et al. An integrative review of factors associated with telomere length and implications for biobehavioral research. Nurs Res. 2014;63:36-50.

17. Shalev I, Entringer S, Wadhwa PD, et al. Stress and telomere biology: a lifespan perspective. Psychoneuroendocrinology. 2013;38:1835-1842.

18. Zacher H, Schmitt A. Work characteristics and occupational well-being: the role of age. Front Psychol. 2016;7:1411.

19. Truxillo DMCDM, Hammer LB. Supporting the aging workforce: a research review and recommendations for workplace intervention research. Annu Rev Organ Psychol Organ Behav. 2015;2:351-381.

20. Peters E, Spanier K, Mohnberg I, Radoschewski FM, Bethge M. Social support as a resource for work ability. Rehabilitation (Stuttg). 2016;55: $102-107$.

21. Rouxel G, Michinov E, Dodeler V. The influence of work characteristics, emotional display rules and affectivity on burnout and job satisfaction: A survey among geriatric care workers. Int J Nurs Stud. 2016;62:81-89.

22. Westermann C, Kozak A, Harling M, Nienhaus A. Burnout intervention studies for inpatient elderly care nursing staff: systematic literature review. Int J Nurs Stud. 2014;51:63-71.

23. Karantzas GC, Mellor D, McCabe MP, Davison TE, Beaton P, Mrkic D Intentions to quit work among care staff working in the aged care sector. Gerontologist. 2012;52:506-516.

24. Kozak A, Kersten M, Schillmoller Z, Nienhaus A. Psychosocial workrelated predictors and consequences of personal burnout among staff working with people with intellectual disabilities. Res Dev Disabil. 2013;34: $102-115$.

25. Maslach C, Jackson SE, Leiter MP. Maslach Burnout Inventory Manual. 3rd ed. Paolo Alto, CA: Consulting Psychologists Press; 1996.

26. Tuomi K, Ilmarinen J, Seitsamo J, et al. Summary of the Finnish research project (1981-1992) to promote the health and work ability of aging workers. Scand J Work Environ Health. 1997;23(suppl):66-71.

27. Mueller A, Weigl M, Heiden B, Glaser J, Angerer P. Promoting work ability and well-being in hospital nursing: the interplay of age, job control, and successful ageing strategies. Work. 2012;41(suppl):5137-5144.

28. Camerino D, Conway PM, Van der Heijden BI, et al. Low-perceived work ability, ageing and intention to leave nursing: a comparison among 10 European countries. J Adv Nurs. 2006;56:542-552.

29. Camerino D, Conway PM, van der Heijden BI, Estryn-Behar M, Costa G, Hasselhorn HM. Age-dependent relationships between work ability, thinking of quitting the job, and actual leaving among Italian nurses: a longitudinal study. Int J Nurs Stud. 2008;45:1645-1659.

30. Ahlstrom L, Grimby-Ekman A, Hagberg M, Dellve L. The work ability index and single-item question: associations with sick leave, symptoms, and health-a prospective study of women on long-term sick leave. Scand J Work Environ Health. 2010;36:404-412.

31. Buessing A, Glaser J. Work Analysis Instrument for Hospitals-Self-Report Version (TAA-KH-S). Göttingen: Hogrefe; 2002.

32. Buessing A, Perrar KM. Burnout measurement. Study of a German version of the Maslach Burnout Inventory (MBI-D). Pflege Zeitschrift. 1994;47(suppl): $20-30$. 
33. Hasselhorn H-M, Freude G. The Work Ability Index. Bremerhaven: Der Wirtschaftsverlag NW; 2007.

34. Cawthon RM. Telomere length measurement by a novel monochrome multiplex quantitative PCR method. Nucleic Acids Res. 2009;37:e21.

35. Tackney J, Cawthon RM, Coxworth JE, Hawkes K. Blood cell telomere lengths and shortening rates of chimpanzee and human females. Am J Hum Biol. 2014;26:452-460.

36. Lucke T. Comparative analysis of biological aging markers in patients with chronic obstructive pulmonary disease (COPD). Institute and Outpatient Clinic for Occupational, Social and Environmental Medicine. Munich: Ludwig-Maximilians-University; 2015.

37. Aubert G, Lansdorp PM. Telomeres and aging. Physiol Rev. 2008;88 557-579.

38. Blackburn EH, Greider CW, Szostak JW. Telomeres and telomerase: the path from maize, Tetrahymena and yeast to human cancer and aging. Nat Med. 2006;12:1133-1138

39. Nilsson PM, Tufvesson H, Leosdottir M, Melander O. Telomeres and cardiovascular disease risk: an update 2013. Transl Res. 2013;162: 371-380.

40. Masi S, D'Aiuto F, Martin-Ruiz C, et al. Rate of telomere shortening and cardiovascular damage: a longitudinal study in the 1946 British Birth Cohort. Eur Heart J. 2014;35:3296-3303.

41. Willeit P, Raschenberger J, Heydon EE, et al. Leucocyte telomere length and risk of type 2 diabetes mellitus: new prospective cohort study and literaturebased meta-analysis. PLoS ONE. 2014;9:e112483.
42. Zhang J, Rane G, Dai X, et al. Ageing and the telomere connection: An intimate relationship with inflammation. Ageing Res Rev. 2016;25:55-69.

43. Bundesministerium fuer Gesundheit. Smoking; 2016. Available at: https://www. bundesgesundheitsministerium.de/service/begriffe-von-a-z/r/rauchen.html. Accessed June 6, 2017.

44. Maslach C, Schaufeli WB, Leiter MP. Job burnout. Annu Rev Psychol. 2001;52:397-422.

45. Stamm TA, Pieber K, Crevenna R, Dorner TE. Impairment in the activities of daily living in older adults with and without osteoporosis, osteoarthritis and chronic back pain: a secondary analysis of population-based health survey data. BMC Musculoskelet Disord. 2016;17:139.

46. Descatha A, Cyr D, Mediouni Z, Goldberg M. Work-related premature ageing: old concept but emerging stakes. Occup Environ Med. 2013;70:675.

47. Podsakoff PM, MacKenzie SB, Lee JY, Podsakoff NP. Common method biases in behavioral research: a critical review of the literature and recommended remedies. J Appl Psychol. 2003;88:879-903.

48. Sanders JL, Newman AB. Telomere length in epidemiology: a biomarker of aging, age-related disease, both, or neither? Epidemiol Rev. 2013;35: $112-131$.

49. Cherkas LF, Aviv A, Valdes AM, et al. The effects of social status on biological aging as measured by white-blood-cell telomere length. Aging Cell. 2006;5:361-365.

50. Oliveira BS, Zunzunegui MV, Quinlan J, Fahmi H, Tu MT, Guerra RO. Systematic review of the association between chronic social stress and telomere length: A life course perspective. Ageing Res Rev. 2016;26:37-52. 